\title{
Dynamics of a Bose-Einstein Condensate on Changing Speeds of an Atomchip Trap Potential
}

\author{
Seung Jin Kim ${ }^{1}$, Jae June Noh', Min Seok Kim ${ }^{1}$, Jin Seung Lee ${ }^{2}$, Hoon Yu ${ }^{1,3}$, and Jung Bog Kim* \\ ${ }^{I}$ Department of Physics Education, Korea National University of Education, Cheongju 363-791, Korea \\ ${ }^{2}$ Agency for Defense Development, Daejon 305-600, Korea \\ ${ }^{3}$ Department of Physics and JILA, University of Colorado, Boulder, Colorado, 80309-0440, USA
}

(Received July 29, 2014 : revised October 21, 2014 : accepted October 21, 2014)

\begin{abstract}
We report experimental behaviors of condensed ${ }^{87} \mathrm{Rb}$ atoms responding to changes in the trap potential of the atomchip. The two-types of adiabatic and non-adiabatic overall changes were implemented by changing the ramp-down speed of the chip-wire current, which can dominantly modify the one-axis magnetic field gradient. Under the adiabatic process, a pure condensate stayed in the initial spin state and collectively oscillated with both monopole and dipole modes, while an atomic cloud above the critical temperature exhibited sound waves in a dense ultracold gas. On the other hand, Bose-Einstein condensate atoms with non-adiabatic perturbation were split into spatially different positions by spin states through spin-flip. We investigated the split ratio among spin states depending on final evaporation frequency. Potential changes, of course, cause collective oscillations regardless of the changing process.
\end{abstract}

Keywords : Collective excitation, Majorana transition, Bose-Einstein condensate, Atomchip OCIS codes : (020.1335) Atom optics; (020.1475) Bose-Einstein condensates; (020.3320) Laser cooling

\section{INTRODUCTION}

Since the first realization of Bose-Einstein condensation in dilute gases confined in a magnetic trap [1, 2], intensive studies of the dynamical behavior of mesoscopic quantum systems have been performed [3-14], and it was expected to explain, for instance, the superfluidity of liquid helium [15]. The study of collective excitations (normal modes) is one of the standard methods used to characterize the behavior of highly-degenerate quantum systems. Since the first measurements of the lowest normal mode frequencies $[3,4]$ verified that the Gross-Pitaevskii equation (GPE) correctly predicted the dynamic behavior of condensate under excitation, the spectroscopy of the excited condensate has been extended to a variety of studies, e.g., frequency shifts $[8,16]$, mode coupling $[8,16,17]$ and damping $[6,18]$.

In the hydrodynamic limit, which is applied to condensates with a large number of atoms, Stringari found the dispersion law for the elementary excitations of a dilute atomic Bose gas confined in both a harmonic trap and repulsive interacting forces [9]. For a cylindrically-symmetric potential,

$$
V_{\text {trap }}(x, y, z)=\frac{1}{2} M \omega_{x}^{2} x^{2}+\frac{1}{2} M \omega_{\perp}^{2}\left(y^{2}+z^{2}\right),
$$

where $M$ is the atomic mass and $\omega_{i}$ is the angular frequency of oscillation along the $i$-axis, the $\mathrm{m}=0$ modes' eigenfrequencies are given by [9]:

$$
\omega_{ \pm}=\sqrt{2 \omega_{\perp}^{2}+\frac{3}{2} \omega_{x}^{2} \pm \frac{1}{2} \sqrt{9 \omega_{x}^{4}-16 \omega_{x}^{2} \omega_{\perp}^{2}+16 \omega_{\perp}^{4}}}
$$

Here - and + signs are to represent monopole and quadrupole mode, respectively. This prediction has been successfully testified by experimental results $[3,4]$.

Since low-lying collective modes have an energy on the order of $\hbar \omega_{i}$ for a harmonically confined Bose-Einstein Condensate (BEC), where $\hbar$ is the Planck constant divided by $2 \pi$, in previous studies collective excitation in a BEC

\footnotetext{
*Corresponding author: jbkim@knue.ac.kr

Color versions of one or more of the figures in this paper are available online.
} 
was performed by applying a series of sinusoidal modulations near the resonance frequency on the confining potential $[3-6,8]$. That is, the energy to excite condensate was provided from external sinusoidal perturbation.

From these previous results, we examined a possibility that a decrease of trap frequency also excites atoms with a contribution of the residual energy since modification of the trap geometry is the basic essential for the dynamical control of the ultracold atoms. The excitation behavior according to the adiabaticity of the deformation is also a topic of our question. For this, the atomchip is a good experimental apparatus because it can easily modify trap geometry at a micrometer scale within a wide range of changing times [19]. In this paper, we report on the observation of the dynamical behaviors of ultracold ${ }^{87} \mathrm{Rb}$ atoms on an atomchip under trap deformation. From the adiabatic deformation, we observed low-lying collective excitations and sound waves with pure condensate and a thermal cloud before the onset of a condensate, respectively. The frequencies were compared with theoretical predictions from the GPE in the hydrodynamic limit and to the sound wave. We were able to separate spatially the condensate into groups by different spin states by Majorana spin-flip through the non-adiabatic deformation. In addition, we investigated the population ratio of the different spin components by varying the final frequency in the $\mathrm{rf}$ evaporation step for the BEC.

\section{EXPERIMENTAL SETUP}

The detailed apparatus and procedures we use for creating the BEC are described elsewhere [20]. In summary, our experimental setup consists of a compact double magnetooptical trap (MOT) apparatus with an anodically bonded atomchip which produces magnetically trapped ${ }^{87} \mathrm{Rb}$ BoseEinstein condensates in the $\mathrm{F}=2, \mathrm{~m}_{\mathrm{F}}=2$ state. An atomic flux of high density is generated from a $2 \mathrm{D}^{+}$MOT in the isolated $2 \mathrm{D}^{+}$MOT chamber [21]. The atoms pass through a $750 \mu \mathrm{m}$ hole and are trapped in a 3D-MOT. The atoms in the 3D-MOT are further cooled below the Doppler limit through a compressed-MOT and polarization gradient cooling. By using an external Z-wire trap, the atoms are transported from the initial 3D-MOT position to near the atomchip surface after optical pumping. About 9 $\times 10^{7}$ atoms can be trapped in an atomchip magnetic trap by the currents through the atomchip wires. After two-step rf forced evaporation, a BEC can be manipulated. Resonant absorption images were taken with the $100 \mu$ s illumination time, and the optical resolution was $3.5 \mu \mathrm{m}$.

The $26 \times 26 \mathrm{~mm}^{2}$ sized atomchip is formed by depositing $10 \mu \mathrm{m}$-thick copper conductor onto a $450 \mu \mathrm{m}$-thick silicon substrate (Fig. 1(a)). The central Z-shaped wire (called the Z-wire, $100 \mu \mathrm{m}$-width) is used with external bias fields to create an Ioffe-Pritchard trap. Higher trap frequency along the $\mathrm{x}$-axis can be obtained with a dimple trap that is created by running a current through the wire that is perpendicular

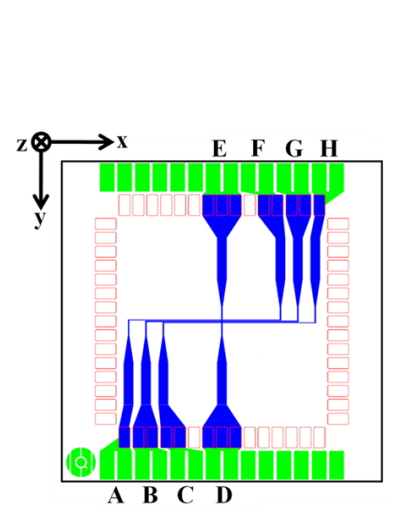

(a)

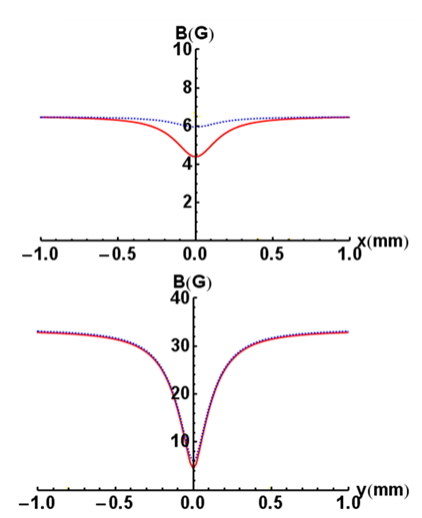

(b)

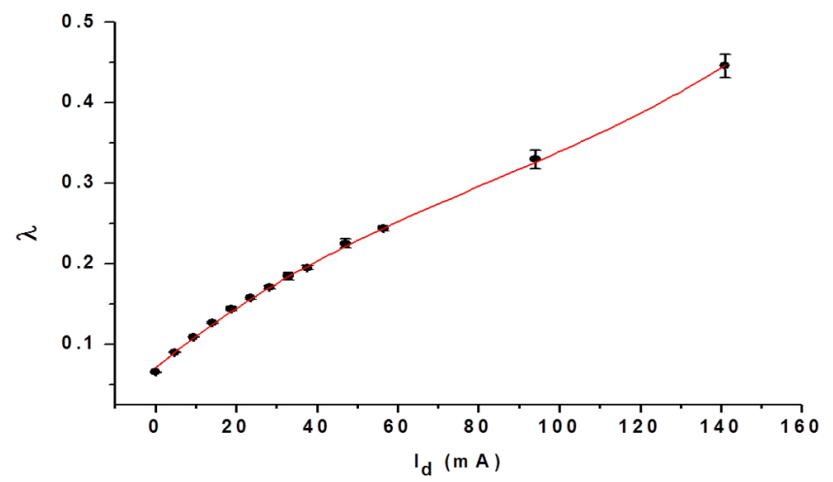

(c)

FIG. 1. (a) Schematic of the atomchip used for magnetic trapping potentials. The central Z-shaped wire (B-G) carries a current and is used together with an external bias field along the $y$-axis to produce an Ioffe-Pritchard trap. The magnetic field gradient along the $\mathrm{X}$-axis can be controlled with the current through the central straight wire (D-E), the dimple wire. (The two Z-shaped wires beside the Z-wire are not used for this study.)

to the Z-wire (called dimple-wire, $100 \mu \mathrm{m}$-width). The two wires beside the Z-wire are designed for an rf-induced potential experiment and are not used for this study. To investigate the motion of the condensate under a trap deformation, the magnetic field of the dimple trap is modified by a ramp-down of the dimple current $I_{d}$. Since the dimple current makes an $\mathrm{x}$-directional field at the center of the trap, the $\mathrm{x}$-directional trap frequency can be changed dominantly with the current (Fig. 1(b)). Fig. 1(c) is the measured anisotropy $\left(\lambda=\omega_{x} / \omega_{\perp}\right)$ of the deformed trap as a function of the final $I_{d}$ in the range of our performance. More reduction of the current makes the trap more anisotropic [22].

For this study, initially, a BEC was prepared after rf forced evaporative cooling within $2.3 \mathrm{~s}$ which is sufficient to damp the atoms' motions induced by the atomic cloud transfer. The prepared BEC typically contains $5.5 \times 10^{4}$ atoms without recognizable thermal fraction and is positioned at $180 \mu \mathrm{m}$ below the atomchip surface with the Z-wire current $\mathrm{I}_{\mathrm{z}}=2.98 \mathrm{~A}$, the $\mathrm{x}$-axis bias field $\mathrm{B}_{\mathrm{x}}=6.86 \mathrm{G}$, the $\mathrm{y}$-axis bias field $\mathrm{B}_{\mathrm{y}}=33.10 \mathrm{G}$ and the $\mathrm{I}_{\mathrm{d}}=0.98 \mathrm{~A}$. 


\section{EXPERIMENTAL RESULTS}

Trap deformation for investigating dynamical behavior was performed in two ways, adiabatic and non-adiabatic changes, by changing the ramp-down duration of dimple current, which is determined by the speed changing trap potential.

\subsection{Adiabatic Changing - Collective Excitations}

For the adiabatic deformation, the dimple current $I_{d}$ was ramped down from $980 \mathrm{~mA}$ to its final value within 10 $\mathrm{ms}$, corresponding to a reduction of the trap frequency. The $10 \mathrm{~ms}$ ramping time was sufficient to guarantee adiabaticity for our experiments because the Larmor frequency at the center of the dimple trap can be estimated as $>2 \mathrm{MHz}$ by the trap bottom $(\sim 700 \mathrm{kHz}$ corresponding to $\sim 1 \mathrm{G})$. After the deformation, the deformed trap was maintained for a variable time, ranging from $0 \mathrm{~ms}$ to $60 \mathrm{~ms}$, to strobe the free dynamical behavior in the deformed trap. The atoms were observed along the y-direction by absorption imaging after a sudden switch off of the magnetic trap and $10 \mathrm{~ms}$ time-of-flight. There was no trap loss during the trap maintenance time over which the delay was varied.

The condensate was excited by the deformation of the trap and two modes of collective excitation were identified from the absorption images: dipole and monopole $\mathrm{m}=0$ modes. The $\mathrm{x}$ and $\mathrm{z}$-directional center-of-mass oscillations (dipole modes) were excited because change of the dimple current displaced the minimum point of the trap slightly due to asymmetry of the trapping Z-wire. From this centerof-mass oscillation, accurate values of trap frequencies can be obtained because dipole mode excitations are not affected by the interatomic forces, nor by the temperature or the statistics [23]. Figure 2(a) shows the shape oscillation of a condensate under the deformation with final $\mathrm{I}_{\mathrm{d}}=47 \mathrm{~mA}$. The condensate exhibited sinusoidal width oscillation with large amplitude along the $\mathrm{x}$-axis. In addition, the $\mathrm{z}$-directional width oscillated with in-phase and relatively small amplitude (Fig. 2(b)). Such width-oscillation behaviors represent monopole $\mathrm{m}=0$ mode (breathing mode) $[9,10]$. Note that the widths were convoluted since the condensate was observed after ballistic expansion. The striations parallel to the z-direction is the optical diffraction fringe formed by the narrowed condensate. The widths of the clouds were extracted from a Thomas-Fermi fit of the absorption profiles along the $\mathrm{x}$ and $\mathrm{z}$-axis at the center. Beyond the optical diffraction limit, which is when the striations occurred, the widths were calibrated from the diffraction spacing. For comparison with the theoretical expectation, the x-directional oscillating widths were fitted with an exponentially decaying sine function, $\sigma(t)=\sigma(0)+A \exp (-t / \tau) \sin (2 \pi(t-\varphi) / T)$, and the frequency of the breathing mode and the decay rate were determined as $127.9 \pm 1.4 \mathrm{~Hz}$ and $28.2 \pm 5.1 \mathrm{~s}^{-1}$, respectively. The expectation value of the breathing mode followed by Eq. (2) was $\omega / 2 \pi=126.4 \mathrm{~Hz}$ because the condensates were in the hydrodynamic limit $N a / a_{H O}=150 \gg 1$ and the trap frequencies of the deformed trap were determined as

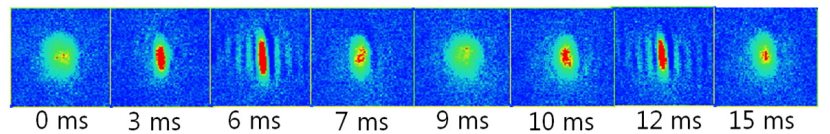

(a)

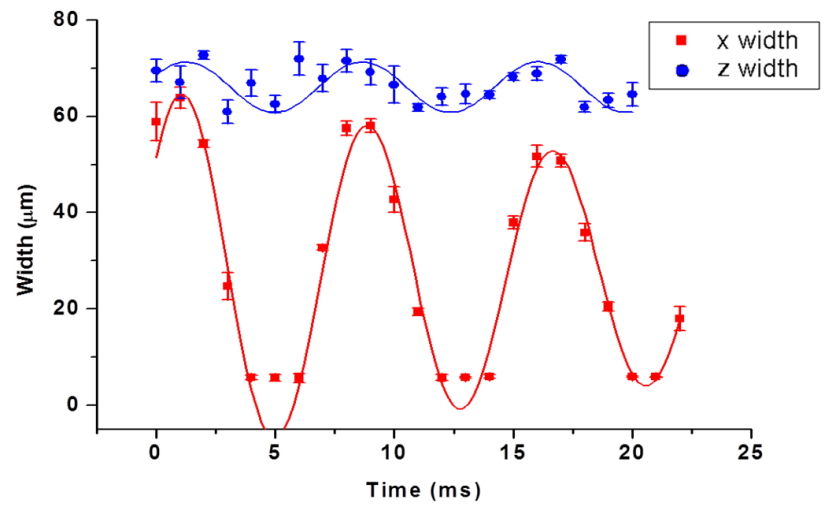

(b)

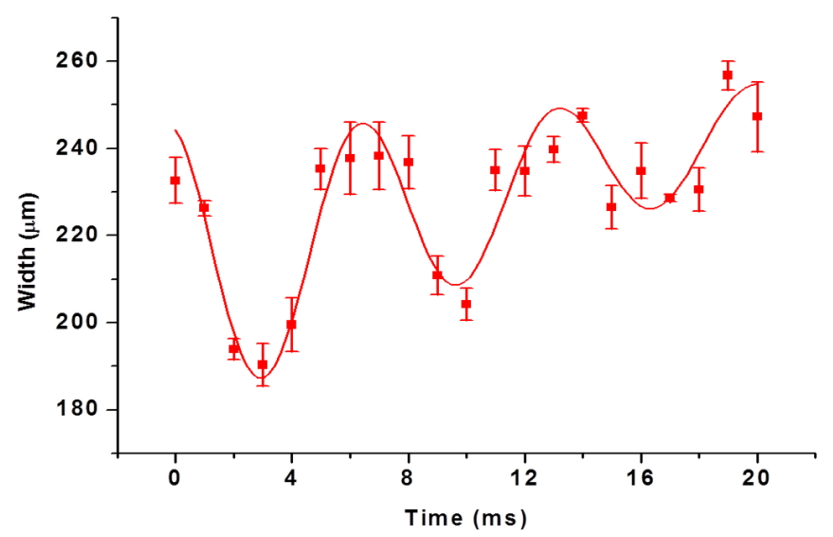

(c)

FIG. 2. The width oscillations induced by the adiabatic deformation with final $I_{d}=47.0 \mathrm{~mA}$. (a) Shape oscillation of a pure BEC. After the deformation, the condensate was allowed to freely oscillate in the deformed trap for a variable time. The absorption images were taken along the $y$-axis after a $10 \mathrm{~ms}$ time-of-flight. Each absorption image has a $360 \times 360$ $\mu \mathrm{m}^{2}$ size and the trap maintenance time below. (b) The widths oscillation of pure BEC. The $\mathrm{x}$ and $\mathrm{z}$-directional widths oscillate in the same phase with large and small amplitudes, respectively: monopole $\mathrm{m}=0$ mode. From fitting an exponentially decaying sine function, the frequency and the damping rate were determined as $127.9 \pm 1.4 \mathrm{~Hz}$ and $28.2 \pm 5.1$ $\mathrm{s}^{-1}$, respectively. (c) The x-directional width oscillation of the thermal cloud at $T / T_{c} \approx 1.5$. The determined frequency is $152.5 \pm 7 \mathrm{~Hz}$ and the width shows rapid diffusion.

$\omega_{x} / 2 \pi=80.2 \pm 1.8 \mathrm{~Hz}$ and $\omega_{\perp} / 2 \pi=367.3 \pm 0.5 \mathrm{~Hz}$ from the dipole mode oscillation. The experimentally determined frequency is consistent with the theoretically expected value within an error bound. The same deformation experiment on a thermal cloud at $T / T_{c} \approx 1.5$ was performed. Fig. 2(c) shows the width oscillation and the frequency was $152.5 \pm 7 \mathrm{~Hz}$. This frequency is also consistent with $2\left(\omega_{x} / 2 \pi\right)=160.4 \pm 3.5$ 
$\mathrm{Hz}$, which is expected for the non-interacting Bose gas [9]. These width oscillations in a thermal cloud can be understood by sound waves. In addition, rapid diffusion of the cloud is identified from the fit line in Fig. 2(c), which indicates the exponentially decaying sine function for accounting for diffusion due to heat, $\sigma(t)=\sqrt{1+H \cdot t} \sigma(0)+A \exp (-t / \tau)$ $\sin (2 \pi(t-\varphi / T)$. We consider the fast diffusion of the cloud is due to the finite depth of the deformed trap and collision induced thermalization of the excitation. From those results, which were consistent with the theoretical expectation, we were able to conclude that the adiabatic deformation of the trap, not by sinusoidal modulation but by ramping, induces collective excitations on the BEC.

After the confirmation of the collective monopole $\mathrm{m}=0$ mode on the condensate, the experiment was done with final $\mathrm{I}_{\mathrm{d}}$ range of 0 to $94 \mathrm{~mA}$. We were not able to include the data with the final $I_{d}$ over $94 \mathrm{~mA}$ because the amplitudes of the dipole mode excitations were too small to determine the trap frequencies. The frequencies of the monopole $\mathrm{m}=$

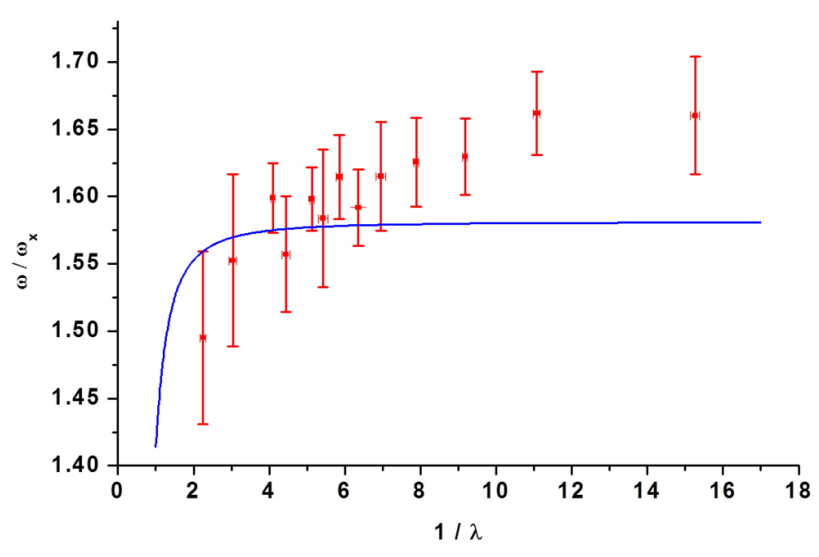

(a)

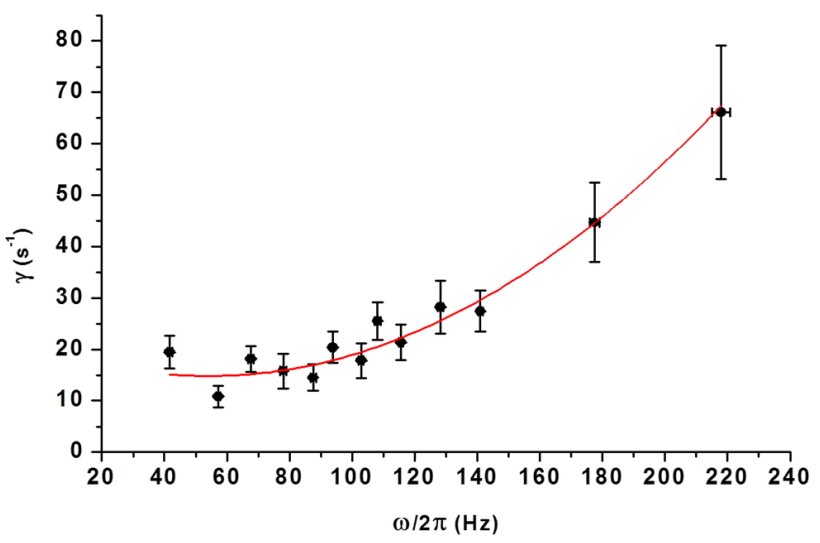

(b)

FIG. 3. (a) Anisotropy-dependent monopole $\mathrm{m}=0$ mode excitation spectrum. The data were obtained from the adiabatic deformation experiments with the final $\mathrm{I}_{\mathrm{d}}$ range of 0 to $94 \mathrm{~mA}$. Solid line indicates theoretical prediction from Eq. (2). (b) The damping rates versus the frequency of monopole $\mathrm{m}=0$ mode excitation. The damping rate quickly decreases with the mode frequency. The solid line is a guide to the eye.
0 mode for various deformed trap geometries were normalized by the corresponding $\mathrm{x}$-directional trap frequency and plotted as a function of the trap anisotropy parameter $1 / \lambda$ as shown in Fig. 3(a). The solid line is the theoretical prediction of the mode frequencies in the hydrodynamic limit as given in Eq. (2). Despite the discrepancy, the measured frequencies demonstrate a similar tendency to the anisotropy and are well below $2\left(\omega_{x} / 2 \pi\right)$ which is expected for noninteracting gases. The mode frequencies higher than the prediction for the highly anisotropic trap can be understood as the effect of anharmonic shift due to the large response amplitudes [5]. Fig. 3(b) presents the decay rate $\gamma$ as a function of the breathing mode frequency. Note that reduction of the final dimple current causes not only a decrease in the mode frequency but also an increase in the response amplitudes. However, we conclude that the decay rate is dominantly affected by the mode frequency because a damping rate of the $m=0$ condensate mode tends to increase with the response amplitude [5]. The damping rate dependent on mode frequency can be understood with the fact that the atom-atom collision rate is proportional to the velocity of the atoms.

\subsection{Nonadiabatic Changing - Majorana Transitions}

To investigate the dynamics under a nonadiabatic deformation, we ramped down the dimple current to $47 \mathrm{~mA}$ within 20 $\mu \mathrm{s}, \sim 50 \mathrm{~T} / \mathrm{s}$, which is sufficiently fast to induce Majorana spin-flips. A variable time delay was introduced between the deformation and the observation of the cloud. The absorption imaging was performed after a $2 \mathrm{~ms}$ time-of-flight. Figure 4(a) is the absorption image taken with a $2.8 \mathrm{~ms}$ time delay and shows the three spatially separated components of ultracold atoms with different spin states: $\mathrm{m}_{\mathrm{F}}=2, \mathrm{~m}_{\mathrm{F}}=1$ and $\mathrm{m}_{\mathrm{F}}=0$. During the deformation, the BEC prepared initially in the $m_{F}=2$ state underwent a fast change in the magnetic field and was spin-flipped by Majorana transition $[24,25]$. After the transition, the spin-flipped $\mathrm{m}_{\mathrm{F}}=0$ component freely fell by gravity while the $\mathrm{m}_{\mathrm{F}}=2$ and the $\mathrm{m}_{\mathrm{F}}=1$ state components oscillated in the deformed trap with different spring constants (Fig. 4(b)). The oscillation frequencies of the two components have a ratio of $\omega_{m_{F}=2} / \omega_{m_{F}=1}=1.43(\simeq$ $\sqrt{2.04})$ which is highly consistent with the expectation $\left(\sqrt{\mu_{m_{F}=2} / \mu_{m_{F}=1}}=\sqrt{2}\right)$. Note that there were no detectable $\mathrm{m}_{\mathrm{F}}=-2$ and $\mathrm{m}_{\mathrm{F}}=-1$ state atoms because they experienced outward forces from the deformed trap and rapidly dissipated. In addition, we investigated the population ratio of the $\mathrm{m}_{\mathrm{F}}$ $=1$ atoms to the sum of the $\mathrm{m}_{\mathrm{F}}=1$ and the $\mathrm{m}_{\mathrm{F}}=2$ atoms as a function of the final frequency used in the $\mathrm{rf}$ evaporation (Fig. 4(c)). Each data point represents 30 measurements. The ratio shows an obvious increasing tendency as the final frequency decreases. For now, we do not have a clear explanation for this. However, from the fact that the population of the $\mathrm{m}_{\mathrm{F}}=2$ state sharply decreases with the frequency while the population of the $\mathrm{m}_{\mathrm{F}}=1$ state remains nearly constant, an inhomogeneous trapping field can be 


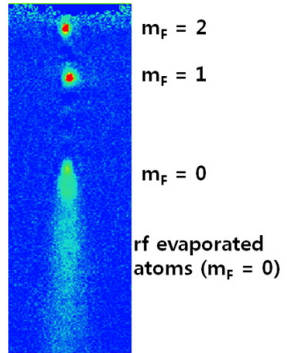

(a)

(c)

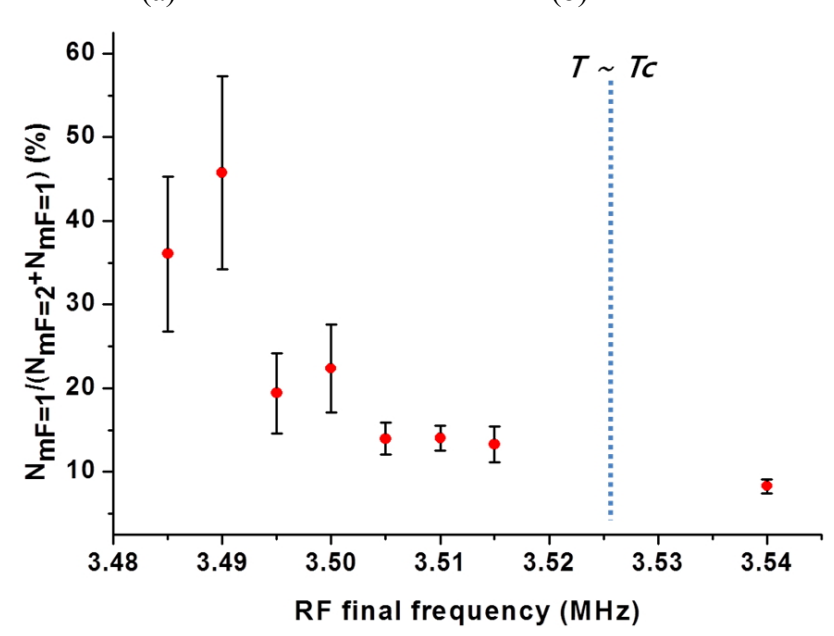

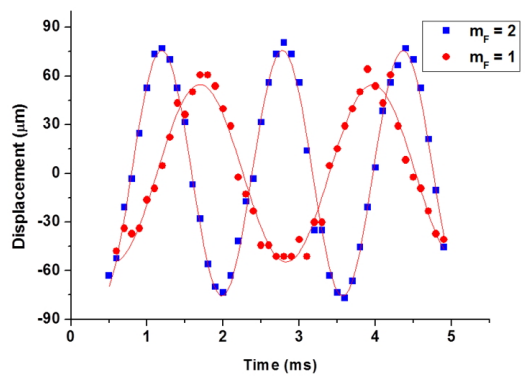

(b)
FIG. 4. Separation of different-spin ultracold atoms under the nonadiabatic deformation. (a) The $2 \mathrm{~ms}$ time-of-flight absorption image with a trap maintaining time of $2.8 \mathrm{~ms}$ after the deformation. Different spin components can be found at different positions since the $\mathrm{m}_{\mathrm{F}}=0$ atoms freely fall by gravity while the $\mathrm{m}_{\mathrm{F}}=1$ and the $\mathrm{m}_{\mathrm{F}}=2$ components oscillate in the deformed trap with different frequencies. (b) The evolution of the dipole mode oscillation with time for the $\mathrm{m}_{\mathrm{F}}$ $=2$ and the $\mathrm{m}_{\mathrm{F}}=1$ atoms. The mode frequency ratio of the $\mathrm{m}_{\mathrm{F}}$ $=2$ to the $\mathrm{m}_{\mathrm{F}}=1$ is $1.43(\approx \sqrt{2.04})$. (c) Population ratio of the spin-flipped $m_{F}=1$ atoms to the sum of the $m_{F}=1$ and the $\mathrm{m}_{\mathrm{F}}=2$ atoms as a function of the final frequency used in the rf evaporation. The vertical dashed line marks the observed transition temperature.

the most plausible reason for the tendency. Some other possible reasons need to be studied, such as temperature dependency of the transition.

\section{SUMMARY AND CONCLUSIONS}

In summary, we studied the dynamical behaviors of ultracold atoms on an atomchip under a trap deformation. With an adiabatic deformation, a BEC shows collective excitations, $\mathrm{m}=0$ monopole and dipole mode oscillations in the deformed trap. The width-oscillation frequency difference between the pure condensate and the non-condensed atomic cloud was clear evidence for the fact that the trap deformation was able to induce collective excitation on the BEC. The measured breathing mode frequencies in the range of the measurements were consistent with the prediction of the mean-field theory in the hydrodynamic limit. Under a nonadiabatic trap deformation that induced a Majorana transition, ultracold atoms were able to be separated spatially due to their trap frequency difference in the deformed trap.

Although collective excitation is not a new topic, the collective behaviors of the BEC on an atomchip is an interesting subject, for example, nonlinear dynamics [26, 27], because the trap geometry can be changed with a high aspect ratio, wide range of time, and high anharmonicity, etc. We believe this data, along with further theoretical study, will help in dynamical control of ultracold atoms on an atomchip.

\section{ACKNOWLEDGMENT}

We are grateful to Prof. Dana. Z. Anderson in JILA for manufacturing the atomchip and the vacuum chamber. This research was supported by a grant to the Atomic Interferometer Research Laboratory for the National Defense funded by DAPA/ADD and by Basic Science Research Program through the National Research Foundation of Korea (NRF) funded by the Ministry of Education (2012R1A1A2041856).

\section{REFERENCES}

1. M. H. Anderson, J. R. Ensher, M. R. Matthews, C. E. Wieman, and E. A. Cornell, "Observation of Bose-Einstein condensation in a dilute atomic vapor," Science 269, 198-201 (1995).

2. K. B. Davis, M. O. Mewes, M. R. Andrews, N. J. van Druten, D. D. Durfee, D. M. Kum, and W. Ketterle, "Bose-Einstein condensation in a gas of sodium atoms," Phys. Rev. Lett. 75, 3969-3973 (1995).

3. D. S. Jin, J. R. Ensher, M. R. Matthews, C. E. Wieman, and E. A. Cornell, "Collective excitations of a Bose-Einstein condensate in a dilute gas," Phys. Rev. Lett. 77, 420-423 (1996).

4. M.-O. Mewes, M. R. Andrews, N. N. van Druten, D. M. Kurn, D. S. Durfee, C. G. Townsend, and W. Ketterle, "Collective excitations of a Bose-Einstein condensate in a magnetic trap," Phys. Rev. Lett. 77, 988-991 (1996).

5. D. S. Jin, M. R. Matthews, J. R. Ensher, C. E. Wiemann, and E. A. Cornell, "Temperature-dependent damping and frequency shifts in collective excitations of a dilute BoseEinstein condensate," Phys. Rev. Lett. 78, $764-767$ (1997).

6. D. M. Stamper-Kurn, H.-J. Miesner, S. Inouye, M. R. Andrews, and W. Ketterle, "Collisionless and hydrodynamic excitations of a Bose-Einstein condensate," Phys. Rev. Lett. 81, 500-503 (1998).

7. O. M. Maragò, S. A. Hopkins, J. Arlt, E. Hodby, G. Hechenblaikner, and C. J. Foot, "Observation of the scissors mode and evidence for superfluidity of a trapped Bose-Einstein condensed gas," Phys. Rev. Lett. 84, 2056-2059 (2000).

8. G. Hechenblaikner, O. M. Maragò, E. Hodby, J. Arlt, S. 
Hopkins, and C. J. Foot, "Observation of harmonic generation and nonlinear coupling in the collective dynamics of a Bose-Einstein condensate," Phys. Rev. Lett. 85, 692-695 (2000).

9. S. Stringari, "Collective excitations of a trapped Bose-condensed gas," Phys. Rev. Lett. 77, 2360-2363 (1996).

10. M. Edwards, P. A. Ruprecht, K. Burnett, R. J. Dodd, and C. W. Clark, "Collective excitations of atomic Bose-Einstein condensates," Phys. Rev. Lett. 77, 1671-1674 (1996).

11. K. G. Singh and D. S. Rokhsar, "Collective excitations of a confined Bose condensate," Phys. Rev. Lett. 77, 1667-1670 (1996).

12. V. M. Pérez-García, H. Michinel, J. I. Cirac, M. Lewenstein, and P. Zoller, "Low energy excitations of a Bose-Einstein condensate: A time-dependent variational analysis," Phys. Rev. Lett. 77, 5320-5323 (1996).

13. V. M. Pérez-García, H. Michinel, J. I. Cirac, M. Lewenstein, and P. Zoller, "Dynamics of Bose-Einstein condensates: Variational solutions of the Gross-Pitaevskii equations," Phys. Rev. A 56, 1424-1432 (1997).

14. L. You, W. Hoston, and M. Lewenstein, "Low-energy excitations of trapped Bose condensates," Phys. Rev. A 55, R1581R1584 (1997).

15. N. N. Bogoliubov, "On the theory of superfluidity," J. Phys. (USSR) 11, 23-32 (1947).

16. F. Dalfovo, C. Minniti, and L. P. Pitaevskii, "Frequency shift and mode coupling in the nonlinear dynamics of a Bose-condensed gas," Phys. Rev. A 56, $4855-4863$ (1997).

17. G. Hechenblaikner, S. A. Morgan, E. Hodby, O. M. Maragò, and C. J. Foot, "Calculation of mode coupling for quadrupole excitations in a Bose-Einstein condensate," Phys. Rev. A 65, 033612 (2002).
18. L. Pitaevskii and S. Stringari, "Landau damping in dilute Bose gases," Phys. Lett. A 235, 398-402 (1997).

19. J. Fortágh and C. Zimmermann, "Magnetic microtraps for ultracold atoms," Rev. Mod. Phys. 79, 235-289 (2007).

20. H. Yu, S. J. Kim, Y. L. Moon, J. S. Lee, and J. B. Kim, "Compact experimental apparatus for producing high-repetitionrate ${ }^{87} \mathrm{Rb}$ Bose-Einstein condensation on atom chip," J. Korean Phys. Soc. 63, 900-906 (2013).

21. K. Dieckmann, R. J. C. Spreeuw, M. Weidemuller, and J. T. M. Walraven, "Two-dimensional magneto-optical trap as a source of slow atoms," Phys. Rev. A 58, 3891-3895 (1998).

22. For the clarity, change of the dimple current induce trap rotation in the $\mathrm{x}-\mathrm{y}$ plane which can be calculated as $\theta_{x y} \approx \arctan \left(I_{d} / I_{z}\right)$. However, there was no noticeable rotational-behavior of the condensate because $I_{d} \ll I_{z}$ and the directions of the imaging are parallel to the rotation plane.

23. C. Cohen-Tannoudji and D. Guéry-Odelin, Advances in Atomic Physics (World Scientific, Singapore, 2011).

24. E. Majorana, "Majorana flops," Nuovo Cimento 9, 43 (1932).

25. I. I. Rabi, "Space quantization in a gyrating magnetic field," Phys. Rev. 51, 652-654 (1937).

26. G.-Q. Li, L.-B. Fu, J.-K. Xue, X.-Z. Chen, and J. Liu, "Collective excitations of a Bose-Einstein condensate in an anharmonic trap," Phys. Rev. A 74, 055601 (2006).

27. H. Ott, J. Fortágh, S. Kraft, A. Günther, D. Komma, and C. Zimmermann, "Nonlinear dynamics of a Bose-Einstein condensate in a magnetic waveguide," Phys. Rev. Lett. 91, 040402 (2003). 